\title{
Influence des changements climatiques sur la distribution géographique de Chrysophyllum albidum G. Don (Sapotaceae) au Benin
}

\author{
François Houéhanou Gbodja GBESSO ${ }^{1 *}$, Brice Hugues Agossou TENTE ${ }^{1}$, \\ Nounagnon Gérard GOUWAKINNOU ${ }^{2}$ et Brice Augustin SINSIN $^{2}$ \\ ${ }^{1}$ Laboratoire de Biogéographie et d'Expertise Environnementale, Faculté des Lettres, \\ Arts et Sciences Humaines, Université d'Abomey-Calavi. BP : 677 Abomey-Calavi, Bénin. \\ ${ }^{2}$ Laboratoire d'Ecologie Appliquée, Faculté des Sciences Agronomiques, \\ Université d'Abomey-Calavi. O1BP 526 Cotonou, Benin. \\ *Auteur correspondant, E-mail :fr2gbesso@yahoo.fr,fr.gbesso@gmail.com \\ Tél : (+229) 97609378/64654345
}

\section{RESUME}

La présente étude a pour objectif principal d'évaluer l'influence des changements climatiques sur la distribution spatiale de Chrysophyllum albidum, une espèce prioritaire et menacée au Bénin. La modélisation de la distribution géographique actuelle et future de l'espèce est basée sur le principe d'entropie maximale (MaxEnt). Elle a été faite à partir des variables bioclimatiques et des points de présence de l'espèce. Deux modèles climatiques ont été utilisés sous le scenario A2 pour la prédiction de la distribution de l'espèce à l'horizon 2050 (les modèles HadCM3 et CSIRO). Les habitats actuellement très favorables à Chrysophyllum albidum correspondent à la zone guinéenne regroupant les phytodistricts de Pobè, du Plateau et de la Vallée de l'Ouémé. Le modèle CSIRO prédit une augmentation considérable de près de 76, $02 \%$ environ des aires actuellement très favorables à l'espèce vers le centre du pays. Par contre, le modèle HadCM3 prédit en 2050 une légère augmentation $(16,27 \%)$ des habitats actuellement très favorables à l'espèce. Ces résultats révèlent que l'habitat favorable de l'espèce est peu menacé par les changements climatiques au Bénin. Ainsi l'espèce a de forte chance de survivre face aux changements climatiques. Ceci représente une opportunité majeure pour sa conservation à long terme.

() 2013 International Formulae Group. All rights reserved.

Mots clés : Changement climatique, modélisation, scénario A2, Chrysophyllum albidum, habitat favorable, Bénin.

\section{INTRODUCTION}

Les Produits Forestiers Non Ligneux (PFNLs) sont d'une importance capitale en termes de leur contribution aux soins de santé, à l'énergie, à l'alimentation, au revenu monétaire et aux autres aspects du bien être humain (Mahapatra et al. 2005). Les populations rurales entretiennent une multitude de relations pratiquement indissociables avec les écosystèmes forestiers à travers les PFNLs en général et les fruitiers autochtones alimentaires en particulier. C'est ainsi qu'au Bénin, 814 espèces médicinales et 128 fruitiers indigènes ont été identifiés comme produits forestiers non ligneux valorisés par les populations rurales (Sinsin et Owolabi, 2001). En Afrique, 25 à $42 \%$ des espèces végétales pourraient être menacées 
d'extinction du fait d'une perte de 81 à $97 \%$ des habitats favorables d'ici 2085 (Boko et al., 2007). D'après les projections, 20 à $30 \%$ des espèces végétales et animales feront face à un plus grand risque d'extinction si le réchauffement global excède $1,5{ }^{\circ} \mathrm{C}$ à $2,5^{\circ} \mathrm{C}$ en Afrique (Busby et al., 2010)

Toutefois, de nos jours, avec les menaces qui pèsent sur la biodiversité, certaines de ces espèces deviennent de plus en plus rares au niveau de leurs habitats naturels. En dehors des menaces anthropiques, le changement climatique apparaît aujourd'hui comme une menace additionnelle pour les populations végétales et animales (Sala et al., 2001).

Le changement du climat constitue une question environnementale qui mérite une attention particulière en matière de planification de la production agricole, de la diversification des productions agricoles et de la conservation des ressources forestières ; étant donné qu'il y a de plus en plus d'évidence que les fluctuations des variables climatiques tels que les précipitations et la température auront une incidence sur la diversité biologique et sur la répartition géographique des habitats favorables aux espèces (GIEC, 2007). Selon les projections, en cas de réchauffement moyen mondial supérieur à $1,5-2,5{ }^{\circ} \mathrm{C}, 20$ à $30 \%$ des espèces végétales et animales seront menacées d'extinction, et des changements majeurs affecteront la structure et le fonctionnement des écosystèmes, les interactions entre espèces, leur distribution géographique, avec des conséquences négatives sur les biens et services associés à ces écosystèmes (GIEC, 2007).

Le consensus scientifique international sur les changements climatiques est sans appel. « Le réchauffement du système climatique est sans équivoque » et, selon une probabilité supérieure à $90 \%$, l'essentiel du réchauffement qui s'est produit depuis 1950 est dû à l'augmentation rapide des concentrations de gaz à effet de serre causée par l'activité humaine (GIEC, 2007). De nouvelles études ont montré que les changements climatiques évoluent plus rapidement que dans les pires scénarios du GIEC. Ces changements climatiques vont avoir de nombreuses répercussions, plus ou moins complexes, sur les arbres. L'augmentation de la concentration atmosphérique en $\mathrm{CO}_{2}$, l'augmentation des températures, les modifications des précipitations, les inondations, les sécheresses fréquentes et de plus longues durées auront des effets significatifs sur la croissance des arbres (Elith et Leathwick, 2009).

Dans un environnement changeant, prédire les variations de distribution d'espèces peut être un enjeu important, notamment en termes de gestion et de domestication de l'espèce. D'autre part, l'évolution récente des techniques statistiques et des systèmes d'informations géographiques permet d'envisager des modèles de distribution beaucoup plus satisfaisants (Elith et Leathwick, 2009). Ces modèles vont d'une part, apporter une meilleure compréhension de l'écologie des espèces et d'autre part permettre des prédictions plus fiables. Parmi ces modèles se trouvent les modèles de Maximum d'Entropie (MaxEnt), (Phillips et al., 2006). Cette méthode présente des caractéristiques intéressantes par rapport à des modèles de type régression par exemple, en lien avec le type de données généralement utilisées. Elle s'avère particulièrement adaptée à des données de présence seulement (qui sont plus courantes en écologie que les données de présence-absence) et à l'utilisation de nombreuses variables en interaction (Elith et al., 2011). Les approches par apprentissage automatique, en particulier MaxEnt, se sont montrées parmi les plus performantes pour la prédiction (Elith et al., 2006 ; Hernandez et al., 2006). Leur usage a toutefois pu être limité en écologie du fait de l'approche corrélative ou «boite noire » pour la prise en compte des variables environnementales dans la probabilité de présence d'une espèce (Elith et Leathwick, 2009), contrairement aux modèles mécanistes (Kearney et Porter, 2009). 
Ceci étant, l'intégration des effets dus aux changements climatiques dans la planification des politiques de conservation apparaît aujourd'hui comme une question plus que jamais nécessaire en matière d'une conservation efficace et durable des ressources génétiques des espèces ligneuses alimentaires prioritaires.

Par ailleurs, l'intégration des espèces fruitières locales à fort potentiel agroforestier a été recommandée comme une solution à l'augmentation croissante de la population et ses implications ultérieures sur la disponibilité et la sécurité alimentaire ainsi que la durabilité environnementale (World Agroforestry Center, 2008). Au Bénin, C. albidum revêt une importance capitale pour les populations locales et a déjà fait l'objet de quelques investigations. En effet, certains travaux au Bénin se sont intéressés à des aspects tels que la structure des populations (Gbesso, 2010), l'utilisation ethnobotanique de l'espèce (Houessou et al., 2012), et son importance socio-économique (Lougbégnon et al., 2012). Bien que les différents organes de la plante tels que les fruits, les feuilles, l'écorce et les racines soient sujets à de multiples usages notamment l'usage alimentaire et médicinal, l'espèce est menacée par un déclin de sa population à cause de la déforestation et la surexploitation des ressources. En addition de ces facteurs et comme souligné plus haut, le changement climatique est apparu comme une contrainte additionnelle à la conservation des ressources phytogénétiques comme $C$. albidum. Il apparait donc urgent d'investiguer les effets probables des changements climatiques sur la distribution géographique de l'espèce afin d'en déduire les habitats favorables à la domestication de l'espèce eu égard aux scenarios climatiques en vue de sa conservation durable.

Ainsi, le présent travail s'est donné comme but d'étudier l'influence du changement climatique sur la distribution géographique de Chrysophyllum albidum au Bénin et de faire ressortir les habitats futurs favorables à la domestication de Chrysophyllum albidum.

\section{MATERIEL ET METHODES \\ Cadre d'étude}

L'étude s'est déroulée dans différentes localités situées dans l'ensemble des districts phytogéographiques du Bénin où des individus de Chrysophyllum albidum ont été enregistrés (Figure 1). Il s'agit des localités des quatre districts phytogéographiques (Côtier, Pobè, Vallée de l'Ouémé et Plateau) de la zone guinéo-congolaise située entre $6^{\circ} 25^{\prime} \mathrm{N}$ et $7^{\circ} 30^{\prime} \mathrm{N}$ et deux districts phytogéographiques (Zou et Bassila) de la zone de transition soudano-guinéenne située entre $7^{\circ} 30^{\prime} \mathrm{N}$ et $9^{\circ} 45^{\prime} \mathrm{N}$ (Figure 1). Ces zones se distinguent les unes des autres non seulement par des conditions environnementales particulières (le climat, les sols, la végétation) mais aussi par les traits humains et groupes socio-culturels variés qui pratiquent plusieurs types d'activités dont l'agriculture, l'élevage, la pêche, la chasse et l'artisanat.

\section{Collecte des données \\ Données d'occurrence}

Plusieurs explorations de terrain ont été organisées dans tout le Bénin. Lors des sorties, on a procédé à l'inventaire systématique des individus de l'espèce dans les différents districts phytogéographiques. Les données de présence ont été prises à l'aide d'un GPS (Global Positioning System). Cette base de données a été complétée par les points de présence disponibles sur le site de Global Biodiversity Information Facility (GBIF) et des travaux antérieurs sur l'espèce (AduBoadu, 2009 ; Lyam et al., 2012).

\section{Variables environnementales}

Les 19 (dix-neuf) variables environnementales ont été obtenues à partir des données brutes de Worldclim (Hijmans et al., 2006) avec une résolution de 2,5 arcminute au sol. Pour les conditions présentes (moyenne sur la période 1950-2000), ces données sont issues d'interpolations à partir 
de données de plus de 20000 stations météorologiques couvrant les 5 continents (Koffi et al., 2008).

Pour les projections climatiques futures, deux modèles de circulation générale (Global Circulation Models : GCMs) parmi les plus recommandés (IPCC, 2007) ont été utilisés : les modèles HadCM3 (Hadley Centre for Coupled Model version 3) et CSIRO (Commonwealth Scientific and Industrial Research Organization).

Les coefficients de corrélation calculés entre les 19 variables ont permis de définir les variables fortement corrélées, deux variables sont fortement corrélées entre elles si le coefficient de corrélation de Pearson est supérieur à 0,7 .

Pour tous ces modèles, les projections faites pour 2050 ont été utilisées sous le scénario d'émission A2. Ce scénario a été utilisé de préférence car il prédit une situation considérée plus probable pour l'Afrique à l'horizon 2050 (Williams et al., 2007). Il décrit un monde très hétérogène, une population à fort taux de croissance avec un faible niveau technologique et de développement.

\section{Analyse des données}

\section{Présentation du modèle de MaxEnt}

Les modèles de niches ont été construits avec le programme MaxEnt (Phillips et al., 2006). MaxEnt utilise une approche statistique appelée entropie maximum pour faire des prévisions à partir de données incomplètes. MaxEnt estime la distribution la plus uniforme/étendue (maximum entropie) des points d'occurrence au sein de la zone d'étude, en tenant compte de la contrainte que, la valeur prévue de chaque variable environnementale de facteur prédictif sous cette distribution estimée, apparie sa moyenne empirique (valeurs moyennes pour le jeu de données d'occurrence). MaxEnt estime la distribution spatiale de l'espèce en fonction de l'entropie maximale de chaque variable environnementale soumise (Phillips et al., 2004, 2006).

\section{Validation du modèle de MaxEnt}

Les variables bioclimatiques ont été soumises à un test de corrélation pour sélectionner celles les moins corrélées ( $\mathrm{r}<$ 0,85) compte tenu des biais que les corrélations ont sur les prédictions futures (Elith et al., 2011). Un test de Jackknife a été ensuite effectué sur les variables bioclimatiques considérées pour déterminer celles qui contribuent le plus à la modélisation. Pour évaluer le modèle, 25\% des points d'observation de l'espèce ont été utilisés pour tester le modèle et $75 \%$ des points ont été utilisés pour calibrer le modèle. La validation croisée du modèle a été répétée cinq fois pour produire des estimations robustes des performances du modèle. La performance du modèle a été évaluée en utilisant la statistique AUC (Area Under the Curve) (Phillips et al., 2006). Un modèle est dit de bonne qualité si la valeur de l'AUC est supérieure à 0,90 (Swets, 1988).

\section{Etapes de la modélisation de niche écologique}

Les étapes de la modélisation de niche écologique favorable sont les suivantes :

- Introduire dans l'algorithme de traitement MaxEnt les coordonnées géographiques de présence de l'espèce en fichier csv (les coordonnées doivent être converties sous forme décimale).

- $\quad$ Intégrer dans le même algorithme les enveloppes bioclimatiques (données sur la précipitation et la température pour un site déterminé dont la plus petite surface prise en considération est de $5 \mathrm{~m}$ x $5 \mathrm{~m}$ ) disponible pour les scenarii du climat de 2000 et de celles prédites de changement climatique en 2050. On entend par scenarii de changement 
climatique les descriptions du futur climat possibles fondées sur des hypothèses à l'égard $\mathrm{du}$ fonctionnement du climat de la terre et des émissions des gaz à effet de serre (GIEC, 2007).

- $\quad$ Evaluer les données intégrées dans l'algorithme MaxEnt qui génèrera après un modèle de niche écologique favorable. La prédiction est obtenue à partir d'interpolation des caractéristiques bioclimatiques de chaque point de présence de l'espèce.

- Ajouter des délimitations géographiques, par exemple le contour du Bénin pour affiner la lecture du modèle.

\section{Cartographie et analyse spatiale}

Les résultats de modélisation produits par MaxEnt ont été importés dans ArcGIS 10 pour cartographier les habitats favorables à l'espèce aussi bien pour les conditions climatiques actuelles que pour les conditions climatiques futures (horizon 2050).

Avec l'outil d'analyse spatiale de ArcGIS, l'étendue de chaque type d'habitat sous des conditions climatiques présentes et futures a été estimée à partir du nombre de pixels occupés par chaque type d'habitat en vue d'évaluer le gain ou la perte dans l'aire favorable à l'espèce à l'échelle du pays (Bénin) suivant les projections climatiques.

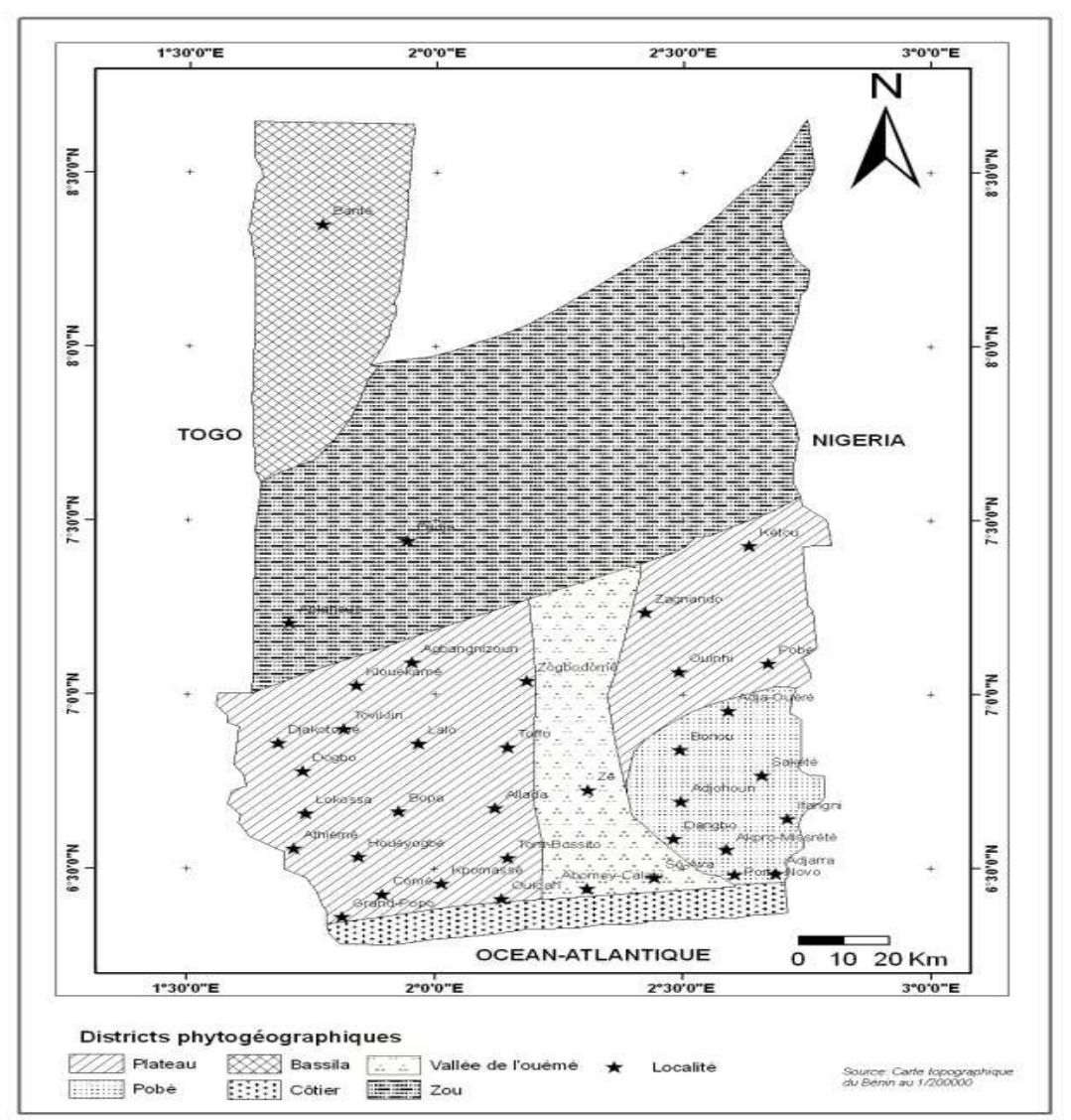

Figure 1 : Situation du cadre d'étude montrant les six districts phytogéographiques abritant $C$. albidum. 


\section{RESULTATS}

Sélection des variables environnementales

$\mathrm{Au}$ total six variables environnementales ont été retenues pour tourner le modèle. Il s'agit de : bio4 (Saisonnalité de la température), bio11 (Température moyenne du trimestre le plus froid), bio12 (Précipitation annuelle), bio14 (Précipitation du mois le plus sec), bio17 (Précipitation du trimestre le plus sec) et bio18 (Précipitation du trimestre le plus chaud). Le Tableau 1 présente la contribution des six variables sélectionnées. De l'analyse de ce tableau il ressort que la variable Bio17 à plus contribuée à l'édification du modèle. Aussi, l'analyse de Jackknife présentée à la Figure 2 a montré que les variables qui ont contribué le plus au développement du modèle lorsqu'elles sont isolément utilisées sont la température saisonnière. Pour chaque variable environnementale, la barre verte montre de combien le gain total est diminuée si cette variable spécifique est exclue de l'analyse. Au contraire, la barre bleue montre le gain obtenu si une variable est utilisée isolément et les variables restantes sont exclues de l'analyse.

Les valeurs de l'AUC sont respectivement de 0,968 et 0,983 , pour la mise en œuvre du modèle MaxEnt et de son test. Ceci suggère une bonne performance de l'algorithme MaxEnt à capturer les variations des données environnementales.

\section{Distribution actuelle et future des habitats favorables à $C$. albidum}

Les résultats de modélisation montrent que globalement, les habitats qui sont actuellement très favorables $\grave{a}$ C. albidum sont principalement localisés dans les phytodistricts du côtier, de Pobè, de la vallée de l'Ouémé et du Plateau alors que les habitats peu favorables ou non sont situés dans les phytodistricts des parties centrale et septentrionale du Bénin correspondant aux domaines soudano-guinéenne et soudanienne (Figure 3).

En effet, au terme de l'analyse des étendues des différents niveaux d'habitats favorables à l'espèce, il apparaît qu'environ $6195,532 \mathrm{~km}^{2}$ (soit 5,4\% du territoire national, îles non comprises) sont actuellement très favorables à $C$. albidum (Tableau 2). Les habitats peu favorable et non favorable occupent respectivement environ $3,01 \%$ et $92,07 \%$ du territoire national (Figure 4).

En ce qui concerne les projections futures à l'horizon 2050, le modèle CSIRO prédit une augmentation considérable de près de $76,02 \%$ environ des aires actuellement très favorables à l'espèce vers le centre du pays. Par contre, le modèle HadCM3 projette en 2050 une légère augmentation $(16,27 \%)$ des habitats actuellement très favorables à l'espèce. Les aires de distribution actuelle et celle prédites sont homogènes quel que soit le scenario utilisé $\left(\right.$ HadCM3 $: \chi^{2}=4,0228, \mathrm{df}=$ 2 , $\mathrm{p}$-value $=0,1338 ;$ CSIRO $: \chi^{2}=1,787, \mathrm{df}$ $=2$, p-value $=0,4092$ ). Il ressort de cette analyse que le changement climatique entrainera une augmentation de la superficie de l'aire de distribution actuelle de $C$. albidum au Bénin. Cette tendance à la hausse des aires de distribution serait due à une augmentation de la pluviométrie prédite par les différents modèles utilisés.

\section{DISCUSSION}

Analyse de la contribution des variables environnementales

Les résultats révèlent que ce sont seulement les variables climatiques qui prédisent les distributions de l'espèce contrairement aux variables biophysiques qui ont une contribution nulle. Cela suppose qu'à l'échelle nationale, la distribution de $C$. albidum est principalement influencée par les facteurs climatiques. Ce résultat semble donc confirmer le fait que les paramètres directs tels que la température, les précipitations sont plus efficaces lorsque la modélisation de la distribution des espèces concerne une large étendue contrairement aux paramètres indirects comme l'altitude, la topographie, le couvert végétal qui sont efficaces pour les petites étendues (Guisan et Zimmermann, 2000). Toutefois, le faible pouvoir prédictif des variables biophysiques peut être également lié à leur agrégation à 2,5 min de résolution, ce qui réduit la quantité de détails qu'elles apportent aux modèles (Guisan et Hofer, 2003). 


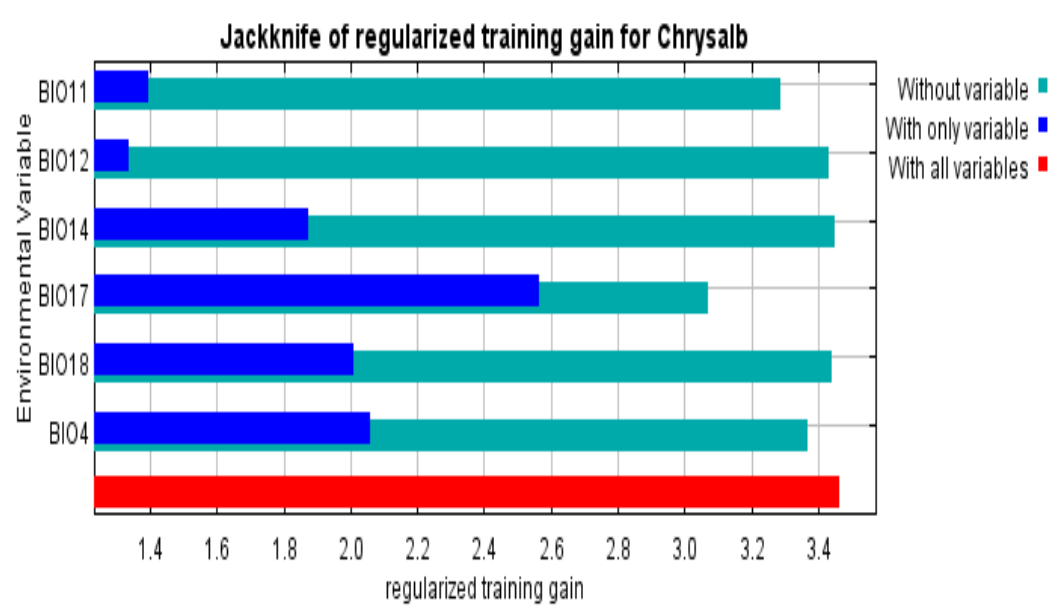

Figure 2 : Résultat du test de Jackknife sur la contribution des modèles
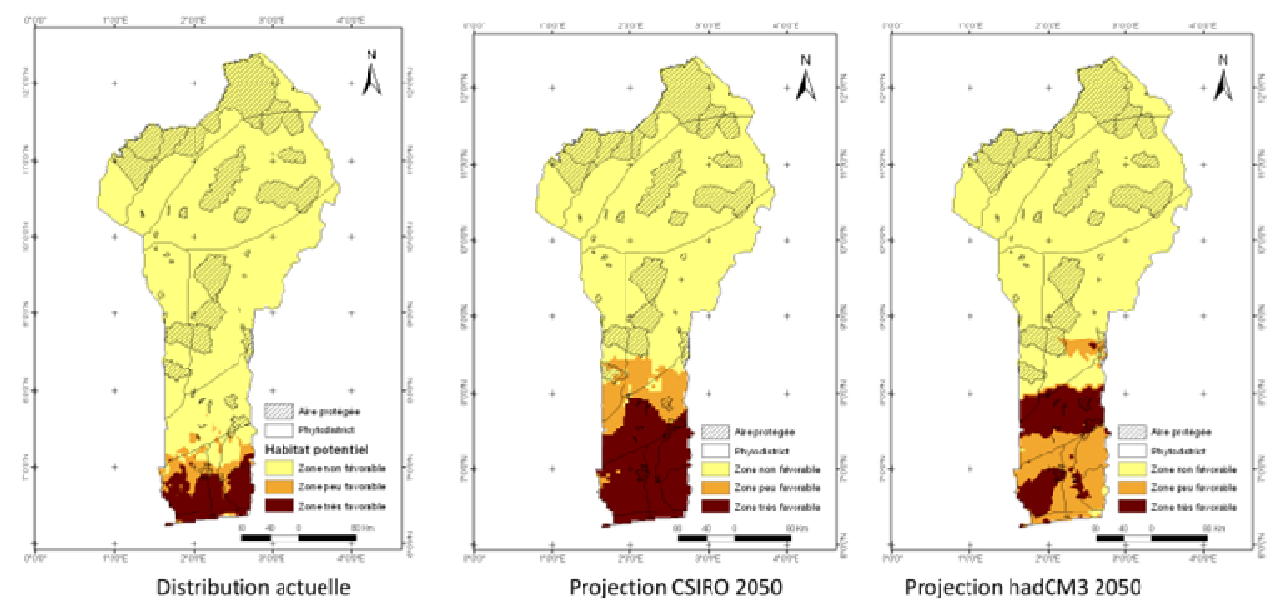

Figure 3 : Distribution potentielle présente et future de C. albidum au Bénin.

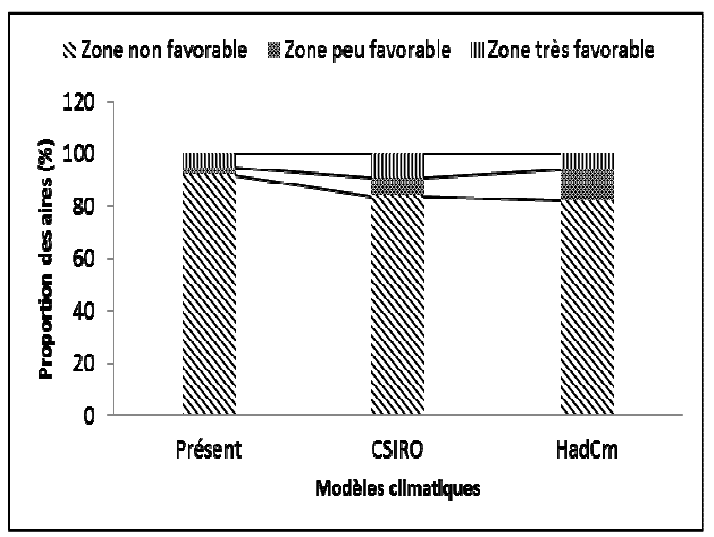

Figure 4 : Etendue des habitats favorables à la conservation de C. albidum au Benin. 
Tableau 1 : Variables bioclimatiques utilisées et leurs contributions au modèle.

\begin{tabular}{lccc}
\hline Code & Variables bioclimatiques & Explication & Contribution (\%) \\
\hline BIO17 & $\begin{array}{c}\text { Précipitations du trimestre le } \\
\text { plus sec }\end{array}$ & $\begin{array}{c}\text { Précipitations totales du trimestre } \\
\text { le plus sec }\end{array}$ & 63,9 \\
\hline BIO4 & Saisonnalité de la température & $\begin{array}{c}\text { Coefficient de variation des } \\
\text { températures }\end{array}$ & 15,5 \\
\hline BIO11 & $\begin{array}{c}\text { Température moyenne du } \\
\text { trimestre le plus froid }\end{array}$ & $\begin{array}{c}\text { Température moyenne du } \\
\text { trimestre le plus froid }\end{array}$ & 9,7 \\
\hline BIO12 & Précipitations annuelles & $\begin{array}{c}\text { Somme de toutes les précipitations } \\
\text { mensuelles estimatives }\end{array}$ & 8,6 \\
\hline BIO14 & Précipitations du mois le plus \\
& sec & $\begin{array}{c}\text { Précipitations totales du mois le } \\
\text { plus sec }\end{array}$ & 1,3 \\
\hline BIO18 & Précipitations du trimestre le \\
& plus chaud & $\begin{array}{c}\text { Précipitations totales du trimestre } \\
\text { le plus chaud }\end{array}$ & 1 \\
\hline
\end{tabular}

Tableau 2 : Variation des habitats favorables à la conservation de C. albidum au Bénin.

\begin{tabular}{lccccccc}
\hline & \multicolumn{2}{c}{$\begin{array}{c}\text { Zone } \\
\text { non favorable }\end{array}$} & \multicolumn{2}{c}{$\begin{array}{c}\text { Zone } \\
\text { peu favorable }\end{array}$} & \multicolumn{2}{c}{$\begin{array}{c}\text { Zone } \\
\text { très favorable }\end{array}$} & $\begin{array}{c}\text { Aire } \\
\text { Totale }\end{array}$ \\
\cline { 2 - 8 } & $\begin{array}{c}\text { Etendue } \\
\left(\mathbf{K m}^{\mathbf{2}}\right)\end{array}$ & $\begin{array}{c}\text { Tendance } \\
(\boldsymbol{\%})\end{array}$ & $\begin{array}{c}\text { Etendue } \\
\left(\mathbf{K m}^{\mathbf{2}}\right)\end{array}$ & $\begin{array}{c}\text { Tendance } \\
(\boldsymbol{\%})\end{array}$ & $\begin{array}{c}\text { Etendue } \\
\left(\mathbf{K m}^{\mathbf{2}}\right)\end{array}$ & $\begin{array}{c}\text { Tendance } \\
(\boldsymbol{\%})\end{array}$ & $\begin{array}{c}\text { Etendue } \\
\left(\mathbf{K m}^{\mathbf{2}}\right)\end{array}$ \\
\hline Présent & 105669,4 & -- & 3457,4172 & -- & 6195,532 & -- & 115322,4 \\
CSIRO & 96804,99 & $-8,39$ & 7581,2403 & $+119,27$ & 10905,2 & $+76,02$ & 115291,4 \\
HadCM3 & 94508,57 & $-10,56$ & 13655,897 & $+294,97$ & 7203,561 & $+16,27$ & 115368 \\
\hline
\end{tabular}

Le signe (-) indique une perte d'habitat favorable et le signe (+) indique un gain. La différence au niveau des aires globales est liée à l'étendue prédite comme non habitat pour l'espèce.

Cependant, même si parmi les variables climatiques, la saisonnalité de la température a montré un pouvoir prédictif très élevé, il est à signaler que ces résultats méritent d'être interprétés avec attention en considérant les caractéristiques écologiques de l'espèce. Ainsi, comme $C$. albidum est une espèce des zones humides marquées par une forte pluviométrie allant de 900 à 3000 mm (DahDovonon, 2002 ; Gbesso, 2010 ; Houessou et al., 2012), on pourrait s'attendre à une forte contribution des variables de précipitation ; ce qui n'est pas le cas puisque les précipitations $\mathrm{du}$ mois le plus sec (bio14) constituent la seule variable de précipitation qui a montré une contribution aux modèles de distribution de l'espèce.

\section{Aire de distribution potentielle de $C$. albidum}

Les projections climatiques sur les périodes à venir indiquent que la taille des surfaces des zones propices au développement de $C$. albidum augmenterait de manière significative au-delà de 2020. L'augmentation de la zone de distribution de $C$. albidum au Bénin à l'horizon 2050 s'expliquerait principalement par l'augmentation projetée de la pluviométrie. Ces résultats sont conformes aux résultats obtenus par Fandohan et al. (2013) sur Tamarindus indica et Gouwakinnou (2011) sur Sclerocarya birrea qui sont des espèces de la zone soudanienne et de Sodé (2013) sur Dialium guineense, une espèce de la même zone que Chrysophyllum albidum. 


\section{Possibilités de conservation de Chrysophyllum albidum}

Nos résultats démontrent qu'aucun modèle climatique n'a prédit un recul total de l'aire de distribution de Chrysophyllum albidum au Bénin. Ces resultats semblent contrastés avec les travaux antérieurs qui annoncent l'impact négatif des changements climatiques sur la diverté biologique (Pearson et al., 2003 ; Thomas et al., 2004). Dans notre cas, le défi de conservation de l'espèce reste mineure quand au chnagement climatique, bien au contraire, nos résultats prédisent une augmentation de l'aire potentielle de l'espèce à l'horizon 2050. La plus grande contrainte quand à la conservation reste les modes d'utilisation des terres. Comme l'a souligné Houessou et al. (2012), la regression de Chrysophyllum albidum au Sud du Bénin est liée en grande partie à la destruction de l'habitat naturel de l'espèce qui se retrouve aujourd'hui confiné dans les systèmes agroforestiers suite à l'urbanisation de plus en plus accélérée de cette partie du Bénin qui est le corollaire d'une croissance démographique galapante. De plus, la dynamique actuelle d'occupation des terres dans le secteur d'étude laisse déjà présager une régression des milieux naturels (vestige de forêt par exemple) au détriment des habitats (Oloukoi, 2006). En définitif, nous concluons que les changements climatiques ne constituent pas une menace à la conservation durable de l'espèce mais plutôt les actions anthropiques. Ainsi, pour augmenter les chances de conservation de l'espèce à long terme, il s'avère important de développer des actions de conservation in-situ et ex-situ de l'espèce dans les systèmes agroforestiers au Sud du Bénin afin de la sauvegarder de la pression de l'occupation des terres suite à l'urbanisation tout azimut dans la région. La conservation de l'espèce dans les jardins botaniques pourrait aussi améliorer l'état de conservation de l'espèce en tant que banque vivante de gènes vivante de $C$. albidum. Toutefois, une grande attention doit être accordée à la variabilité génétique de l'espèce dans sa stratégie de conservation au sein des systèmes agroforestiers ou jardins botaniques afin d'accroitre sa capacité à faire face aux changements dans le temps.

\section{Implications de l'étude pour la conservation durable de $C$. albidum}

La présente étude fait ressortir que les zones actuellement favorables à la culture de l'espèce au Bénin sont principalement localisées dans la zone guinéenne.

Quel que soit le modèle, les zones du territoire national ainsi que le réseau d'aires protégées situées dans la zone guinéenne resteront toujours très favorables à la culture et à la conservation de C. albidum en 2050. Par conséquent c'est cette zone qui semble être susceptible de fournir à $C$. albidum des conditions climatiques adéquates pour sa culture et sa conservation sans incidences majeures face aux effets du changement climatique à l'horizon 2050. Dans cette considération, comme l'espèce a un fort potentiel agroforestier, son utilisation dans les exploitations agricoles à travers des systèmes agroforestiers au sein de la zone concernée serait d'une importance capitale en termes de contribution aux revenus des populations locales.

Toutefois, les zones peu favorables pourraient être prises en compte pour la culture et la conservation de l'espèce dans le futur à condition que des écotypes aux régions plus ou moins arides soit génétiquement sélectionné. Dans ces conditions, même en cas de diminution des précipitations associée à l'augmentation de la température, ces zones qui sont susceptibles de devenir des zones moyennement favorables à l'espèce en 2050 (modèle HadCM3) pourraient dans une certaine mesure continuer à offrir les conditions favorables à la survie de ces nouveaux écotypes. Dans cette optique, la variabilité génétique de l'espèce en termes d'adaptation à la sécheresse et de croissance 
de l'arbre serait également déterminante pour une meilleure domestication.

\section{Conclusion}

Le changement climatique constitue l'un des principaux facteurs contribuant à l'altération des structures globales de la biodiversité en exerçant des changements dans la distribution spatiale des espèces. C'est pour évaluer l'importance de tels changements sur les fruitiers autochtones que la présente étude a essayé de fournir quelques informations utiles sur la distribution de Chrysophyllum albidum. Ces informations pourront servir à l'élaboration des stratégies de conservation de l'espèce et à l'amélioration des capacités adaptatives des populations locales afin de réduire leur vulnérabilité aux effets du changement climatique. La zone guinéenne associée aux agrosystèmes continuera à assurer la culture et la conservation de l'espèce sous le climat futur (horizon 2050). Toutefois, d'autres facteurs influençant la distribution des espèces tels que les interactions biotiques, l'adaptation génétique et les capacités de dispersion de l'espèce doivent être prises en compte afin de tirer des conclusions pertinentes devant permettre de mieux orienter les prises de décision en matière de conservation.

\section{REMERCIEMENTS}

Les auteurs remercient le Ministère de l'Enseignement Supérieur et de la Recherche Scientifique (MESRS) pour l'appui financier dont a bénéficié F. GBESSO dans le cadre de sa Thèse. Ils remercient aussi Dr. Laurent G. HOUESSOU, Dr. Toussaint O. LOUGBEGNON et tous les lecteurs qui ont contribué à l'amélioration de la qualité scientifique du manuscrit.

\section{REFERENCES}

Adu-Boadu M. 2009. Evaluation of the agroforestry potential of Chrysophyllum albidum in the Akuapem North District. Msc thesis. College of Agriculture and
Natural Resources. Kwame Nkrumah University of Science and Technology.

Boko M, Niang I, Nyong A, Vogel C. 2007. Africa. Climate change 2007: Impacts, Adaptation and Vulnerability. Contribution of Working Group II to the 4th Assessment report of the Intergovernmental Panel on Climate Change. Cambridge University Press : Cambridge, UK; 433-467.

Busby JW, Smith TG, White KL, Strange SM. 2010. Locating Climate Insecurity: Where are the Most Vulnerable Places in Africa? University of Texas, The Robert Strauss Center for International Security and Law, Climate Change and African Political Stability (CCAPS): Austin, TX, USA.

Dah-Dovonon JZ. 2002. Rapport du Bénin. In Réseau «Espèces Ligneuses Alimentaires ». Compte rendu de la première réunion du Réseau: 11-13 décembre 2000. Edited by Eyog- Matig O, Gaoué OG, Dossou B. IPGRI: Ouagadougou, Burkina Faso, 2-19.

Elith J, Graham CH, Anderson RP, Dudı kM, Ferrier S, Guisan A, Hijmans, R J, Huettmann F, Leathwick JR, Lehmann ALiJ, Lohmann LG, Loiselle BA, Manion G, Moritz C, Nakamura M, Nakazawa Y, Overton, JMcC, Peterson AT, Phillips SJ, Richardson KS, Scachetti-Pereira R, Schapire RE, Soberon J, Williams S, Wisz MS, Zimmermann, NE. 2006. Novel methods improve prediction of species' distributions from occurrence data. Ecography, 29: 129-151.

Elith J, Phillips SJ, Hastie T, Dudík M, Chee YE, Yates CJ, 2011. A statistical explanation of MaxEnt for ecologists. Diversity and Distributions, 17: 43-57.

Elith J, Leathwick JR. 2009. Species Distribution Models: Ecological Explanation and Prediction Across Space and Time. Annual Review of Ecology Evolution and Systematics, 40: 677-697. 
Fandohan B, Gouwakinnou G, Fonton NH, Sinsin B, Liu J. 2013. Impact des changements climatiques sur la répartition géographique des aires favorables à la culture et à la conservation des fruitiers sous-utilisés : cas du tamarinier au Bénin. Biotechnol. Agron. Soc. Environ. (in press).

Gbesso GHF. 2010. Structure, dynamique des populations et importances socioéconomiques et culturelles de Chrysophyllum albidum sur le plateau d'Allada (Sud Bénin). Mémoire de DEA, Université d'Abomey-Calavi, AbomeyCalavi, p. 87.

GIEC 2007. Bilan 2007 des changements climatiques: Contribution des Groupes de travail I, II et III au quatrième rapport d'évaluation du GIEC. [Équipe de rédaction principale, Pachauri R.K. et Reisinger A.]. GIEC, Genève, Suisse, p. 103.

Gouwakinnou NG, 2011. Population ecology, uses and conservation of Sclerocarya birrea (A. Rich) Hocchst. (Anacardiaceae) in Benin, West Africa. $\mathrm{PhD}$ Thesis, University of Abomey Calavi, Abomey-Calavi, p. 176.

Guisan A, Zimmermann NE. 2000. Predictive habitat distribution models in ecology. Ecol. Modell., 135: 147-186.

Guisan A, Hofer U. 2003. Predicting reptile distributions at the mesoscale: relation to climate and topography. Journ. Biogeogr., 30: 1233-1243.

Hernandez PA, Graham CH, Master LL, Albert DL. 2006. The Effect of Sample Size and Species Characteristics on Performance of Different Species Distribution Modeling, IPCC (2000). IPCC Special Report on Emissions Scenarios. Cambridge: UK, Cambridge University.

Hijmans RJ, Graham CH. 2006. The ability of climate envelope models to predict the effect of climate change on species distributions. Glob. Chang. Biol., 12: 110.

Houessou GL, Lougbegnon OT, Gbesso GHF, Anagonou SEL, Sinsin B. 2012. Ethnobotanical study of the African star apple (Chrysophyllum albidum G. Don) in the Southern Benin (West Africa). Journal of Ethnobiology and Ethnomedicine, 8: 40.

IPCC. 2007. Climate Change: Synthesis Report. Cambridge University Press: New York, USA.

Kearney M, Porter W. 2009. Mechanistic niche modelling: combining physiological and spatial data to predict species ranges. Ecology Letters, 12: 1-17.

Koffi KJ, Boyemba F, Ndjele M, Mate M, Robbrecht E, Lejoly J, Bogaert J. 2008. La distribution spatiale des Acanthaceae dans les secteurs phytogéographiques de Ndjele (1988) en R.D. Congo. Annales de la Faculté des Sciences de Kisangani, 13: $1-8$.

Lougbegnon OT, Anagonou L, Houessou GL., Gbesso GHF, Sinsin B. 2012. Importance socioéconomique des organes (fruits, écorces et racines) de la pomme étoile blanche (Chrysophyllum albidum G. Don, 1831) sur le plateau d'Allada. Ann. Univ. de Lomé, sèr. Lett., Tome XXXI-1, 294: 157-165.

Lyam PT, Adeyemi TO, Ogundipe O. 2012. Distribution Modeling of Chrysophyllum albidum G.Don in South-West Nigeria. Nat Env Sci., 3(2): 7-14.

Mahapatra AK, Albers HJ, Robinson EJZ. 2005. The impact of NTFP sales on rural household's cash income in India's dry deciduous forest. Environmental Management 35(3): 258-265.

Oloukoi J. 2006. Dynamique de l'occupation du sol dans le département des collines et impact sur l'utilisation des bas-fonds. Mémoire de DEA, Université d'Abomey-Calavi, Abomey-Calavi, p. 84.

Pearson RG, Dawson TP. 2003. Predicting the impacts of climate change on the distribution of species: are bioclimate 
envelope models useful? Global Ecology and Biogeography, 12: 361-371.

Phillips SJ, Dudik M, Schapire RE. 2004. A maximum entropy approach to species distribution modeling. In: Proc. of the 21st International Conference on Machine Learning, Banff, Canada.

Phillips SJ, Anderson RP, Schapired RE. 2006. Maximum Entropy Modeling of species geographic distributions. Ecological Modelling, 190: 231-259.

Sala E, Ballesteros E, Starr RM. 2001. Rapid decline of Nassau grouper spawning aggregations in Belize: Fishery Management and Conservation Needs, 26: 23-30.

Sinsin B, Owolabi L. 2001. Monographie nationale de la diversité biologique. Rapport de synthèse. Ministère de l'Environnement, de l'Habitat et de l'urbanisme (MEHU), Cotonou, Bénin, p. 41.

Sodé I. 2013. Influence du changement climatique sur la distribution géographique des fruitiers autochtones alimentaires : cas de Dialium guineense Willd. (Leguminosae-Caesalpinioideae) au Bénin. Mémoire de Licence Professionnelle, Université d'AbomeyCalavi, ENSAT/Kétou, p.71.

Swets JA. 1988. Measuring the accuracy of diagnostic systems. Science, 240: 12851293.

Thomas CD, Cameron A, Green RE, Bakkenes M, Beaumont LJ, Collingham YC, Erasmus BFN, de Siqueira MF, Grainger A, Hannah L, Hughes L, Huntley B, van Jaarsveld AS, Midgley GF, Miles L, Ortega-Huerta MA, Townsend Peterson A, Phillips OL, Williams SE. 2004. Extinction risk from climate change. Nature, 427: 145-148.

Williams JW, Jackson ST, Kutsbach JE. 2007. Projected distribution of novel and disappearing climates by 2100 AD. Proc. Natl. Acad. Sci. U.S.A., 104: 5738-5742.

World Agroforestry Centre. 2008. Transforming Lives and Landscapes. Strategy 2008-2015. World Agroforestry Centre: Nairobi; 39. 